Recepción: 01 / 03/ 2018

Ciencias técnicas y aplicadas

Aceptación: 15 / 05 / 2018

Artículo de investigación

Publicación: 07 / 08 / 2018

\title{
Evaluación de riesgos mecánicos en área de mecanizado con método fine para prevenir accidentes
}

Evaluation mechanics hazards in machining area with the fine method to prevent accidents

\begin{abstract}
Avaliação de riscos mecânicos na área de usinagem com um método fino para evitar acidentes
\end{abstract}

\footnotetext{
Marcial A. Montero-Fierro I

marcial.monterof@ug.edu.ec

Luis A. Vela-Albuja ${ }^{\text {II }}$

luis.velaa@ug.edu.ec

Roberto O. Arevalo-Moscoso III

roberto.arevalom@ug.edu.ec

César J. Reyes-Chamaidan ${ }^{\text {IV }}$

cesar.jr@gmail.com
}

Correspondencia: marcial.monterof@ug.edu.ec

\footnotetext{
I. Docente de la Universidad de Guayaquil, Guayaquil, Ecuador.

II. Docente de la Universidad de Guayaquil, Guayaquil, Ecuador.

III. Docente de la Universidad de Guayaquil, Guayaquil, Ecuador.

IV. Graduado de la Universidad de Guayaquil, Guayaquil, Ecuador.
} 


\section{Resumen}

El presente trabajo está basado en la evaluación de los riesgos mecánicos que existen en el área de mecanizado, debido a las diversos accidentes como cortes, golpes y atrapamiento, se realizó la matriz triple criterio para identificar los riesgos y con el método FINE que es una herramienta para evaluar cuantitativamente accidentes laborales, el cual permitió determinar que la inadecuada capacitación sobre el uso de equipos de protección personal y operación de maquinarias (fresadora, torno, recitadora, esmeril) y en el rebabeado de las piezas revestidas influye en la problemática, se pretende dar diversas alternativas de solución; A) Capacitaciones sobre Seguridad Industrial y Salud Ocupacional; B) Capacitaciones sobre la prevención de riesgos mecánicos y usos de los EPP; C) la compra de los EPP de acuerdo a las actividades realizadas en el área de tal manera que se tomarán como soluciones las alternativas B y C las cuales tendrán un costo de \$4,705.57. Las actividades deben llevarse a cabo en base de leyes y reglamentos vigentes para la protección de la integridad física de los trabajadores evitando lesiones y muerte por accidentes, lo cual es beneficioso para los procesos productivos y economía de la empresa.

Palabras claves: Riesgos mecánicos, Método W. Fine, Cortes en extremidades, Mecanizado, Matriz triple criterio, Capacitaciones. 


\section{Abstract}

The present thesis is based in the evaluation of mechanical hazard that exist in the machining area, due to several accidents as cuts, blows and trappings, we did the Triple-Criterion Matrix to identify the risks and using the FINE method which is a theory to evaluate occupational accidents quantitatively, which allowed To determine that inadequate training on the use of personal protective equipment and operation of machinery (milling machine, lathe, reciter, Emery) and in the deburring of the coated parts influences the problematic, it is intended to give various alternatives of Solution; (A) Industrial Safety and occupational health training; (B) training on the prevention of mechanical risks and uses of PPE; (C) The purchase of the EPP according to the activities carried out in the area in such a way that the alternatives B and $C$ which will cost $\$ 4,705.57$, will be taken as solutions. The activities must be carried out on the basis of laws and regulations in force for the protection of the physical integrity of the workers avoiding injuries and death by accidents, which is beneficial for the productive processes and economy of the company.

Keywords: Mechanical hazards, W. Fine method, Limb cuts, Machining, Triple-criterion matrix, Trainings. 


\section{Introducción.}

La edad media fue una de las épocas que promovieron el desarrollo de diversos tipos de maquinarias, entre ellas está el torno mecánico que en sus inicios se utilizaba mediante un pedal con palanca que impulsaba su funcionamiento. Los tornos sirven para dar forma, taladrar, pulir y realizar otras operaciones concretas, las cuales son sumamente utilizadas en la industria desde siglos pasados. (Ulloa-Enríquez, 2012)

En el siglo XVI, los tornos ya se propulsaban de forma continua mediante manivelas o energía hidráulica; los tornos mecánicos al comenzar la revolución industrial en Inglaterra durante el siglo XVII, surgieron tornos capaces de dar forma a una pieza metálica, en el siglo XVII, el torno pesado industrial para la producción en serie de piezas de precisión, en el año 1780, Jacques de Vaucanson construye un torno con portaherramientas deslizantes, en los años siguientes 1797 , Henry Maudslay y 1820 Thomas Blanchard, fue creado el torno con revolver. (Ruiz Hernández , 2011) (DÍAZ ZAZO, 2015)

A finales del siglo XIX, estos tipos de torno eran fabricados en distintos tamaños y pesos. El diseño y patente en 1890 de la caja de Norton, incorporada a los tornos paralelos, dio solución al torno manual de engranaje. El torno control numérico $(\mathrm{CN})$ es automatizado programable. Se diseñó para adaptar variaciones en la configuración de los productos, es una forma de automatización programable que es controlado a través de números, letras y otros símbolos que están codificados en un formato apropiado para definir un programa de instrucciones para desarrollar una tarea concreta. (Díaz Moline, 2007)

Entre los tipos de tornos que actualmente se utilizan en la industria del mecanizado, cuya aplicación depende de la cantidad de piezas a mecanizar, y de la complejidad de las piezas y 
durezas: torno horizontal, torno copiador, torno revolver, torno automático y torno vertical. (Beltrán Molina, López Becerra, \& Murcia Chinchilla, 2015)

El objetivo de la presentes investigación es evaluar los riesgos mecánicos en el área de mecanizado utilizando el método FINE para prevenir accidentes.

\section{Metodología.}

\section{Situación actual}

Las maquinarias que posee el área de mecanizado son cuatro: tornos paralelos, tres medianos y uno grande tiene una fresadora de torreta, una rectificadora planeadora que con tiene una base metálica para asentar las piezas a rectificar, tiene un esmeril de banco. El área contiene un extractor de aire axial de 17 pulgadas de cuatro paletas.

En el torno paralelo grande es el único donde se puede dar medidas exactas al maquinar y rectificar los trabajos realizados debido a que las bancadas del mismo están sin desgastes al contrario de los otros tres tornos paralelos que poseen un desgaste en las bancadas por esa razón al no dejan las medidas requeridas y simplemente se deja a sobremedida para luego dar medidas exactas en el torno paralelo grande. La limpieza de maquinarias donde se ha realizado el trabajo es inusual y la presencia de residuos sólidos es lo que pueden causar riesgos en los trabajadores. 


\begin{tabular}{|l|c|}
\hline \multicolumn{2}{|c|}{ Área de mecanizado } \\
\hline $\begin{array}{l}\text { Espacio donde se recibe } \\
\text { los trabajos por realizarse }\end{array}$ & $18,21 \mathrm{~m} 2$ \\
\hline Maquinarias & $45,24 \mathrm{~m} 2$ \\
\hline Oficinas de taller & $5,00 \mathrm{~m} 2$ \\
\hline Bodega de herramientas & $3,50 \mathrm{~m} 2$ \\
\hline Total & $71,95 \mathrm{~m} 2$ \\
\hline
\end{tabular}

Tabla No. 1.- Dimenciones área de mecánizado

\section{Política de Seguridad Industrial}

La gerencia está gestionando la implementación de una política en base a las leyes y reglamento vigentes en la seguridad industrial para la protección de la integridad física de los trabajadores, para evitar lesiones y muerte por accidentes también para la protección de maquinarias y materiales de planta; que ayude a proteger la economía de la empresa y de sus procesos productivos.

\section{Compromiso Institucional con la Seguridad Industrial}

El compromiso de gerencia ha permitido gestionar la implementación de una política de seguridad y brindar el apoyo a los colaboradores en caso de accidentes laborales al igual que cada trabajador tiene conocimiento del compromiso y la responsabilidad que debe impartir al momento de iniciar cada una de las tareas para el maquinado de los rodillos barnizadores cumpliendo con el respectivo uso de los EPP para prevenir accidentes.

\section{Prevención de Riesgo Laboral}

Para la prevención de riesgos laborales, se evaluará el puesto de trabajo e identificará mediante la matriz triple criterio a que riesgos están expuestos los trabajadores, una vez 
Marcial A. Montero-Fierro; Luis A. Vela-Albuja; Roberto O. Arevalo-Moscoso; César J. Reyes-Chamaidan

identificados los riesgos se los analizará mediante el método FINE para definir el grado de peligrosidad y realizar medidas preventivas para reducir o eliminar los riesgos.

\section{Recursos Productivos}

Se inicia con el pedido de rodillos barnizadores, de impresión los mismos que llegan con desgaste y partidura en el revestimiento, también la fabricación y revestimiento de piezas especiales, después de recibir la orden de fabricación del departamento de ventas para su revestimiento se procede a desbastar y dejar limpio el rodillo para darle las características requeridas por el cliente y luego ser revestidas de poliuretano. También se realizan trabajos de barras, planchas, acoples flexibles, aros soportadores de carga, aros de montacarga (llantas) y el revestimiento de piezas especiales (retenedores de sistemas hidráulicos y neumáticos, zapatas, ruedas krusher estas ruedas son utilizadas en las industrias que fabrican cajas de cartón). Los trabajos de piezas especiales se realizan mediante moldes que se fabrican según muestra o características requeridas por el cliente y ser revestidos de poliuretano.

\section{Procesos}

Los procesos que se realizan en el área del mecanizado es el maquinado de rodillos barnizadores. Al recibir los rodillos se procede a tomar medidas y características en que llegan. Luego se empieza a desbastar para dejarlos limpio y después se traslada a darle el tratamiento de arenado, llega el rodillo nuevamente a la empresa para ser revestido y luego se traslada al área de mecanizado para maquinarlo con cuchillas cobalto, luego se procede a darle una rectificada rápida (45min) y la rectificada final (4 horas) luego de ser rectificado se procede a lijar (lijas 100, $220 \mathrm{y}$ 360 de agua) y con la ayuda de talco luego se empaqueta con papel y cartón después se los ubica en producto terminado. 
El revestimiento de piezas especiales se empieza a fabricar el molde de acuerdo con la muestra o plano solicitado por el cliente, se procede a revestir luego de ser revestido se traslada al área de mecanizado para ser rebabeadas (cuchillos) y limpiadas para después proceder a maquinar con cuchillas cobalto (redonda y en punta) y darle medidas según el plano requerido por el cliente y se empaqueta.

Los aros o ruedas de montacargas después de ser llevadas al área de mecanizado se fabrica un centrador metálico de acuerdo la medida del interior de la rueda para maquinarlas con cuchillas cobalto, se procede a realizarse un chaflan de $45^{\circ}$ de cada extremo de la rueda y se procede a lijar (100 de fierro) para dejarlas terminadas y empaquetarlas.

\section{Mapa de Procesos}

El mapa de procesos se realiza con la finalidad de describir en secuencias los procesos del área de mecanizado, un mapa de procesos es una representación gráfica que debería estar presente en una organización, según Cabo Salvador (2014) los procesos dependen de las actividades propuestas las cuales interactúan mostrando la relación entre ellos y sus relaciones con el exterior para cumplir objetivos propuestos.

Es una herramienta estratégica, que permite a la empresa tener la dirección, planificación y tomar decisiones; Los procesos operativos son los propios del área que se rigen a las necesidades de los clientes, esto permite planificar las actividades; los procesos de apoyo, son los que proporcionan recursos necesarios que sirven para complementar los procesos claves de la organización, que se someten a una evaluación constante sobre el control de calidad lo que permite tener un trabajo bien hecho. A continuación, se muestra el mapa de procesos con su respectiva descripción. 
Marcial A. Montero-Fierro; Luis A. Vela-Albuja; Roberto O. Arevalo-Moscoso; César J. Reyes-Chamaidan

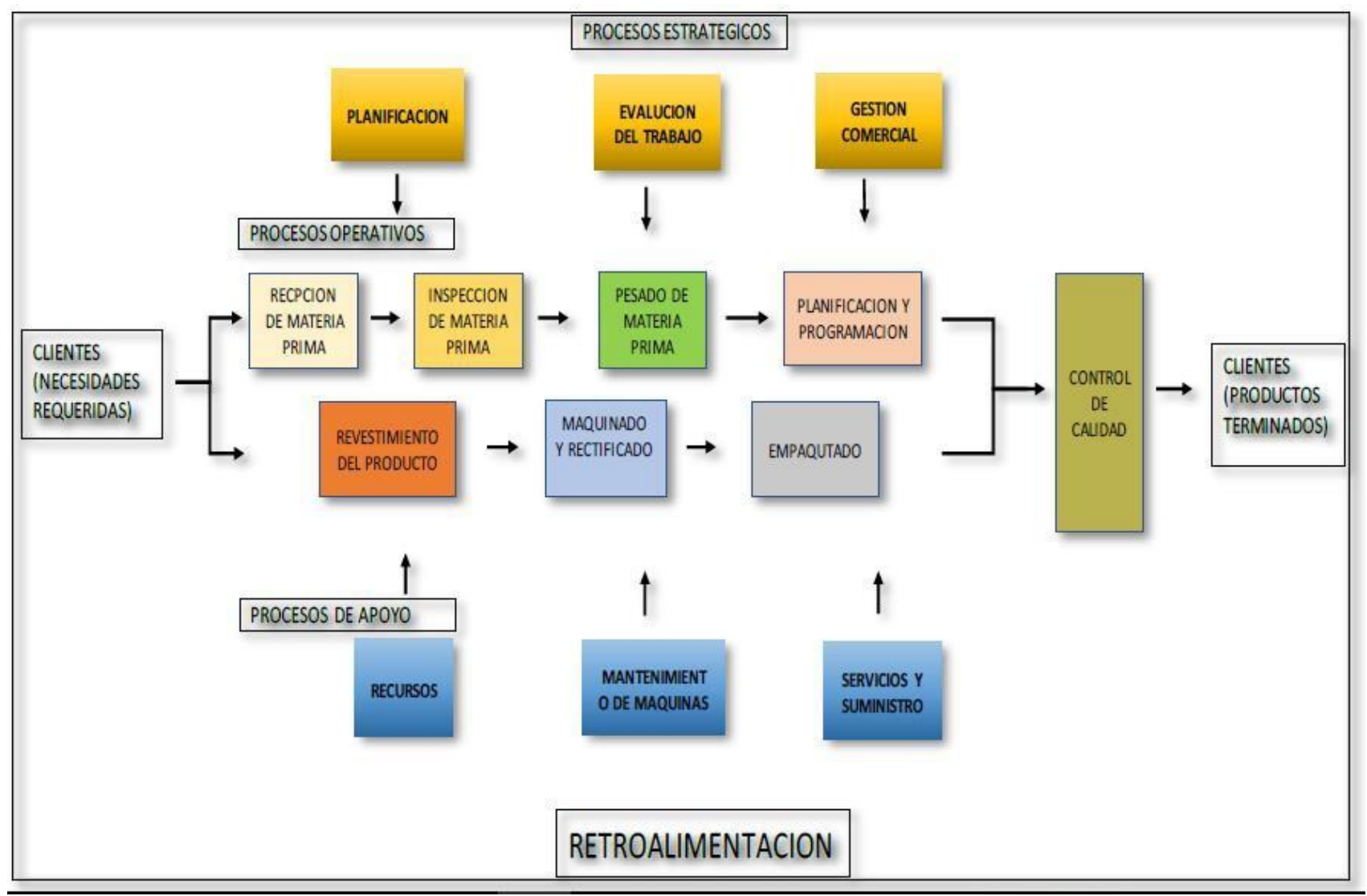

Figura No. 1.- Mapa de procesos

\section{Procesos Operativos}

Los procesos productivos es una secuencia de actividades a seguir, de todos los procesos de los productos maquinado en el área de mecanizado se van a describir las actividades en el maquinado de piezas especiales y de los aros de montacargas (llantas) debido a que serán analizados los riesgos existentes en las actividades del producto:

- Se saca la pieza revestida del horno y se deja enfriando.

- Se verifica que la pieza revestida este en buen estado, que su revestimiento no tenga burbujas.

- Luego de verificar se traslada la pieza al área de mecanizado para ser maquinadas. 
- Se empieza a rebabear la pieza revestida quitando el desperdicio de material.

- Debido a que son piezas especiales se procede a realizar un centrador de acuerdo a las características de la pieza.

- Se ubican cuchillas (cobalto) para proceder al maquinado de la pieza.

- Luego de maquinar con cuchilla (cobalto) se la rectifica dependiendo la dureza de la pieza se le una rectificada rápida.

- Luego se da la rectificada final dejando la medida requerida.

- Luego se lija con lija de agua (100,220 y 320) para dejar un acabado exitoso en el producto.

- Se verifica que el trabajo esté terminado y maquinado de acuerdos a las medidas requeridas.

- Luego de ser verificado se lo traslada a productos terminado.

Estas actividades nombradas son realizadas solo en el maquinado de piezas especiales.

En este proceso se fabrica cualquier tipo de partes y piezas especiales. Por ser el Poliuretano un material fundible, se puede obtener piezas que no están disponibles en el mercado, esto lo conseguimos a partir del plano, de la muestra de la pieza o del molde a fabricar.

Por lo tanto, después de este proceso de la fabricación del molde y de revestimiento se toma los procesos operativos del maquinado detallados anteriormente con cada una de las actividades realizadas tomando en cuenta las inspecciones necesarias para continuar con la actividad siguiente de acuerdo al proceso.

Cabe recalcar que también se puede realizar la construcción del molde de la pieza indicada. Con el material que se posee se logra la fabricación de acoples, retenedores de sistemas hidráulicos, y neumáticos, etc. 
Marcial A. Montero-Fierro; Luis A. Vela-Albuja; Roberto O. Arevalo-Moscoso; César J. Reyes-Chamaidan

\section{Diagrama de flujo de procesos operativos}

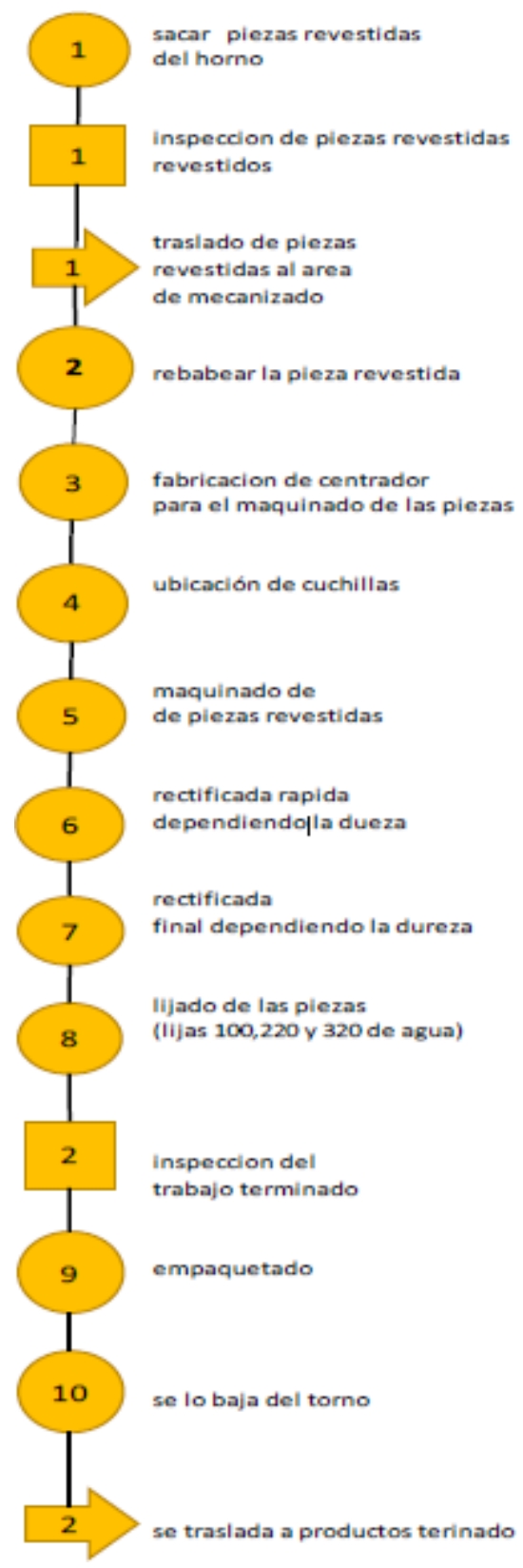

Diagrama $N^{\circ}$. 1.- Diagrama de flujo de procesos operativos 


\section{Riesgos Laborales: Físicos, Químicos, Biológicos, Mecánicos, Ergonómicos, Psicosociales}

En el área se identificaron los siguientes riegos:

- Riesgos Físicos: Ruido, vibración y ventilación insuficiente.

- Riesgos Químicos: Polvo (poliuretano rectificado)

- Riesgos Biológicos: Presencia de vectores (cucarachas) y agentes biológicos.

- Riesgos Mecánicos: Desorden, manejo de las herramientas cortantes y punzantes, espacios reducidos, caída de objetos en manipulación, golpes, obstáculos en el piso, piso irregular y trabajos de mantenimiento.

- Riesgos Ergonómicos: Esfuerzo físico, levantamiento manual de objetos, posición forzada de pie.

- Riesgos Psicosocial: Alta responsabilidad, turnos rotativos.

\section{Factores de riesgos laborales}

Los riesgos mecánicos se identificaron y dieron como resultado ser los que más incidencia en el área de mecanizado tuvieron, sobre todo en el proceso del maquinado de piezas especiales y aros de montacargas (llantas).

- Desorden

- Manejo de las herramientas cortantes

- Manejo de herramientas punzantes (rebabeadores)

- Espacios reducidos

- Caídas de objetos en manipulación

- Golpes 
- Mal apilamiento de objetos

- Choques contra objetos, etc.

\section{Registro de Problemas}

En el área de mecanizado se han registrado cortes en extremidades superiores con herramientas cortantes y punzantes, golpes por la mala manipulación, además el desorden y la limpieza de las máquinas obstaculizan el paso en el área del trabajador, son problemas que suceden frecuentemente al realizar este tipo de trabajo; con el análisis de estas causas se propone tomar ciertas medidas preventivas para evitar ciertos accidentes laborales, los accidentes más frecuentes son los cortes en los cuales se va a centralizar y dar medidas preventivas se clasificarán en dos clases:

- Cortes leves: Heridas de poca profundidad que son ocasionadas por las herramientas o piezas con filos cortantes.

- Cortes graves: Heridas con mayor profundidad que pueden afectar tejidos profundos y darse una sutura de herida abierta.

Estos cortes sondados en máquinas como tornos con las cuchillas de maquinado en la inadecuada manipulación, en fresadora debido a la limpieza de la fresa en movimiento de rotación, en el rebabeado de piezas revestidas con cuchillos rebabeadores. 
Evaluación de riesgos mecánicos en área de mecanizado con método fine para prevenir accidentes

\begin{tabular}{|l|c|c|c|c|c|}
\hline & Año 2016 & 2017 & total & $\% / 2016$ & $\% / 2017$ \\
\hline Cortes leves & 260 & 120 & 380 & $68 \%$ & $32 \%$ \\
\hline Cortes moderados & 40 & 18 & 58 & $69 \%$ & $31 \%$ \\
\hline cortes graves & 8 & 3 & 11 & $73 \%$ & $27 \%$ \\
\hline
\end{tabular}

Tabla No. 2.- Porcentaje de accidentes de cortes y golpes por año

Identificación de problemas. Diagrama Causa- Efecto. 5M Maquinaria, Medio Ambiente, Materiales, Métodos y Mano de obra)

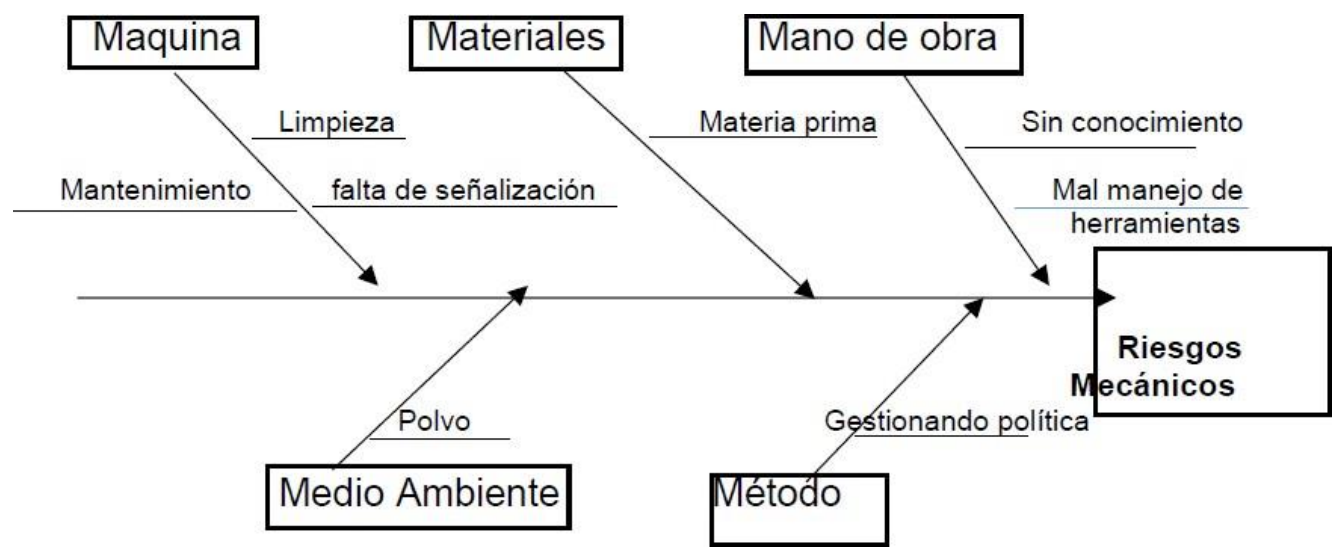

\section{Diagrama $N^{\circ}$ 2.- Identificación del problema}

Análisis de los problemas

En el área de mecanizado los riesgos con más incidencia son los riesgos mecánicos que se presentan frecuentemente en el maquinado de piezas especiales y aros de montacargas (llantas) al realizar la limpieza y rebabeado del revestimiento. El problema se analizará mediante el método WILIAM FINE. 


\section{Clasificación de las actividades de trabajo por puesto de trabajo}

En el área de mecanizado es el puesto de trabajo que se va a analizar, se detalla las siguientes actividades.

- Se traslada las piezas revestidas al área de mecanizado.

- Proceder a limpiar y a rebabear el material que queda en las piezas después de ser revestidas.

- Fabricar centrador para que acople en las piezas revestidas y aros de montacargas.

- Ubicación de cuchillas y ajuste a la torre del torno.

- Se empieza a maquinar con cuchillas dejando exceso para poder rectificar y realizar diseño deseado por el cliente o según la muestra.

- Dar rectificada rápida dependiendo la dureza $(45$ - 50, 60 - 65, 70 - 75, 80 - 85) shore "A" que ha sido revestida la pieza especial, Los aros de montacargas (llantas) son revestidas de dureza 92 shore "A"

- A los aros de montacargas se le fabrica un chaflan de $45^{\circ}$ por cada extremo.

- Se empieza a lijar el diámetro para luego ser empaquetado.

\section{Análisis de Riesgo por puesto de trabajo}

Se analizará el área de mecanizado donde los trabajadores se exponen a riesgos mecánicos frecuentemente por lo que va a demostrarse mediante la matriz de riesgos triple criterio describiendo las actividades realizadas que ocasionan a que el trabajador exponga su salud en el área de trabajo.

Selección de actividades a realizar en el puesto de trabajo

El trabajo que se va analizar es el maquinado de productos fabricados por la empresa entre los trabajos realizados, se tienen en cuenta las piezas especiales (retenedores de sistemas hidráulicos, 
zapatas ruedas krusher, difusores etc.) y en los aros de montacargas (llantas); en el área de mecanizado se analizará los riesgos mecánicos a que se exponen los trabajadores y que ponen en riesgo su salud e integridad física, y mediante el análisis se deben tomar medidas de prevención y el uso de equipos de protección personal de acuerdo con el trabajo que se esté realizando en el área de mecanizado.

\section{Identificar los riesgos de accidentes potenciales}

Para demostrar que el riesgo de accidente potencial es el riesgo mecánico, se utilizará la matriz de riesgo triple criterio para analizar y darle valoración a cada actividad realizada por el trabajador y establecer que existe peligrosidad en la realización del trabajo y después del análisis obtener como resultado que el riesgo potencial es el maquinado de productos fabricados por la empresa.

La matriz de identificación de riesgos, es una herramienta de gestión que permite identificar peligros y evaluar los riesgos asociados a los procesos de cualquier organización. Se entiende por peligro cualquier acto o situación que puede derivar en hechos negativos en el lugar de trabajo. A su vez, el riesgo es la combinación de la probabilidad de que se materialice un peligro y de las consecuencias que puede implicar. La matriz de riesgos es una herramienta esencial para la empresa, supone un elemento en el que se encuentran todos los peligros significativos de accidentes de trabajo y enfermedades laborales. Permite a las organizaciones identificar, evaluar y controlar de un modo permanente los riesgos de accidentes y enfermedades del trabajo. La matriz de riesgos se muestra a continuación, en la cual se tienen en cuenta riesgos mecánicos, físicos, químicos, biológicos, ergonómicos, psicosociales. 
Es sumamente importante elaborar esta matriz para demostrar que el riesgo de accidente potencial es el riesgo mecánico, mediante la matriz de riesgo triple criterio para analizar y valorar cada actividad que realizan todos y cada uno de los trabajadores del área de mecanizado de la organización para así establecer que existe peligrosidad en la realización del trabajo y posteriormente en base a los resultados establecer el riesgo potencial con el fin de mitigarlo.

\section{Resultados.}

\begin{tabular}{|l|c|c|c|c|c|}
\cline { 2 - 5 } \multicolumn{1}{c|}{} & MODERADO & IMPORTANTE & INTOLERABLE & TOTAL & \multicolumn{1}{c}{} \\
\hline FISICOS & 7 & 0 & 0 & 7 & $13 \%$ \\
\hline MACANICOS & 6 & 10 & 2 & 18 & $35 \%$ \\
\hline QUIMICOS & 1 & 6 & 0 & 7 & $13 \%$ \\
\hline BIOLOGICOS & 0 & 0 & 0 & 0 & $0 \%$ \\
\hline ERGONOMICOS & 11 & 0 & 0 & 11 & $21 \%$ \\
\hline PSICOSOCIALES & 6 & 3 & 0 & 9 & $17 \%$ \\
\hline & 31 & 19 & 2 & 52 & $100 \%$ \\
\hline $\begin{array}{l}\text { ESTIMACIÓN } \\
\text { TOTAL }\end{array}$ & $60 \%$ & $37 \%$ & $4 \%$ & & \\
\end{tabular}

En el análisis hecho en la matriz de riesgo triple criterio se analizó los sietes riesgos más importantes donde se demostró que el mayor riesgo en el área de mecanizado es el riesgo mecánico y que las actividades que pueden causar peligros son los cortes al rebabear el producto y al maquinarlo debido al mal manejo de herramientas cortantes y punzantes, se evaluara y tomara medidas preventivas para reducir los accidentes.

También se reflejan otros riesgos eminentes en el área de mecanizado que representan un porcentaje menor; riesgos físicos y químicos representan el 13\%, clasificados en moderada e 
Evaluación de riesgos mecánicos en área de mecanizado con método fine para prevenir accidentes

importante que compromete un riesgo mínimo que se pueden prevenir a corto plazo; los factores psicosociales representan un $17 \%$, esto está estrechamente relacionado con el problema a tratar, debido a que los trabajadores al sufrir alguna lesión o accidente laboral afecta de forma biopsicosocial y los ergonómicos con un $21 \%$ en moderado estos resultados identificados por la matriz triple criterio da a conocer a que riesgo debemos prevenir inmediatamente el cual fue el riesgo mecánico ya mencionado.

Valoración de los riesgos por puesto de trabajo: Determinar sí los riesgos son tolerables

\section{Factores de Riesgo}

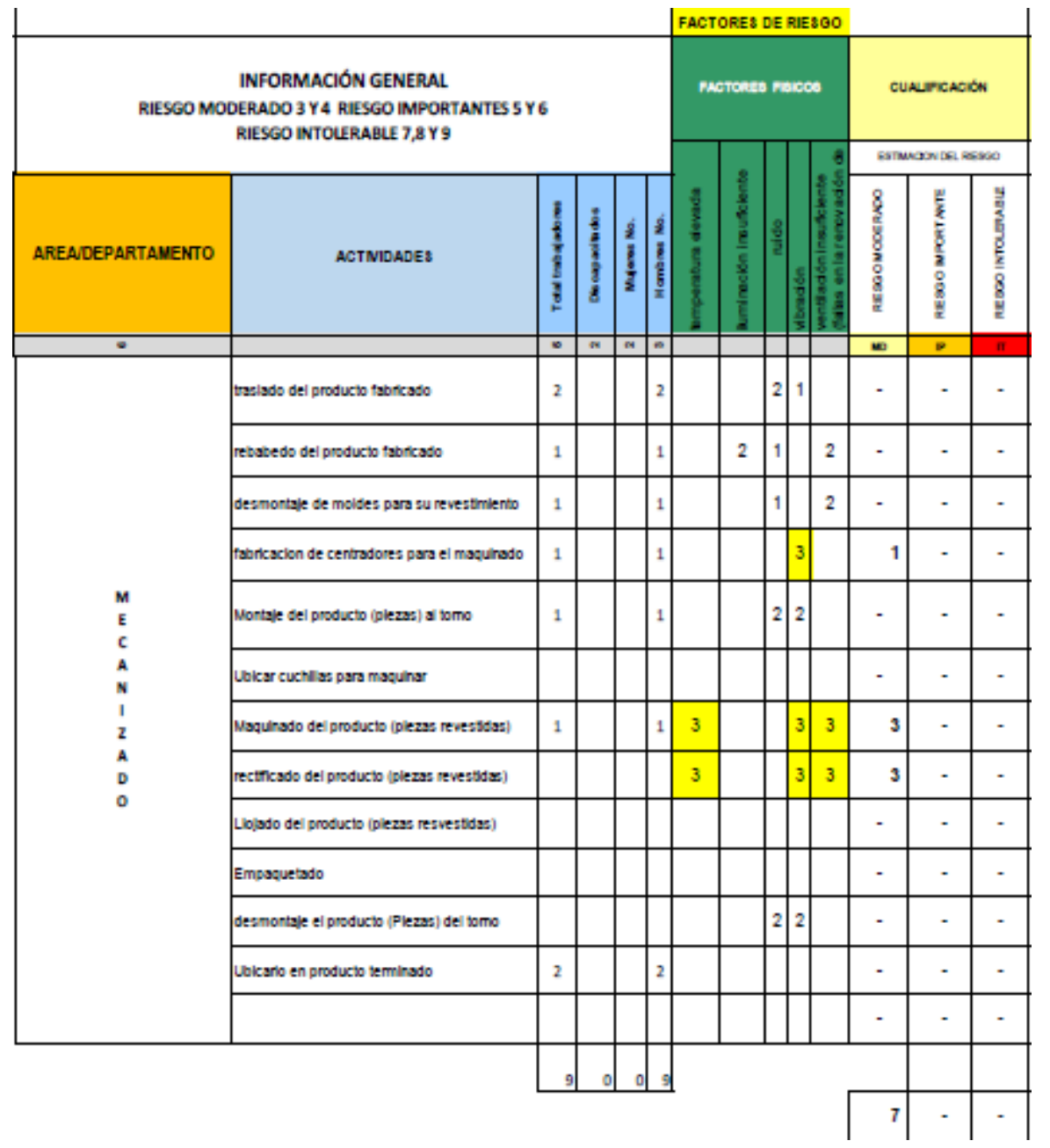


Se analizaron los riesgos físicos en las actividades realizadas en el área de mecanizado se evalúa según el nivel de riesgo se identificaron siete riesgos moderados en las actividades realizadas por temperatura, vibración y ventilación insuficiente en el área donde se deberá corregir estos factores.

\section{Riesgos Mecánicos}

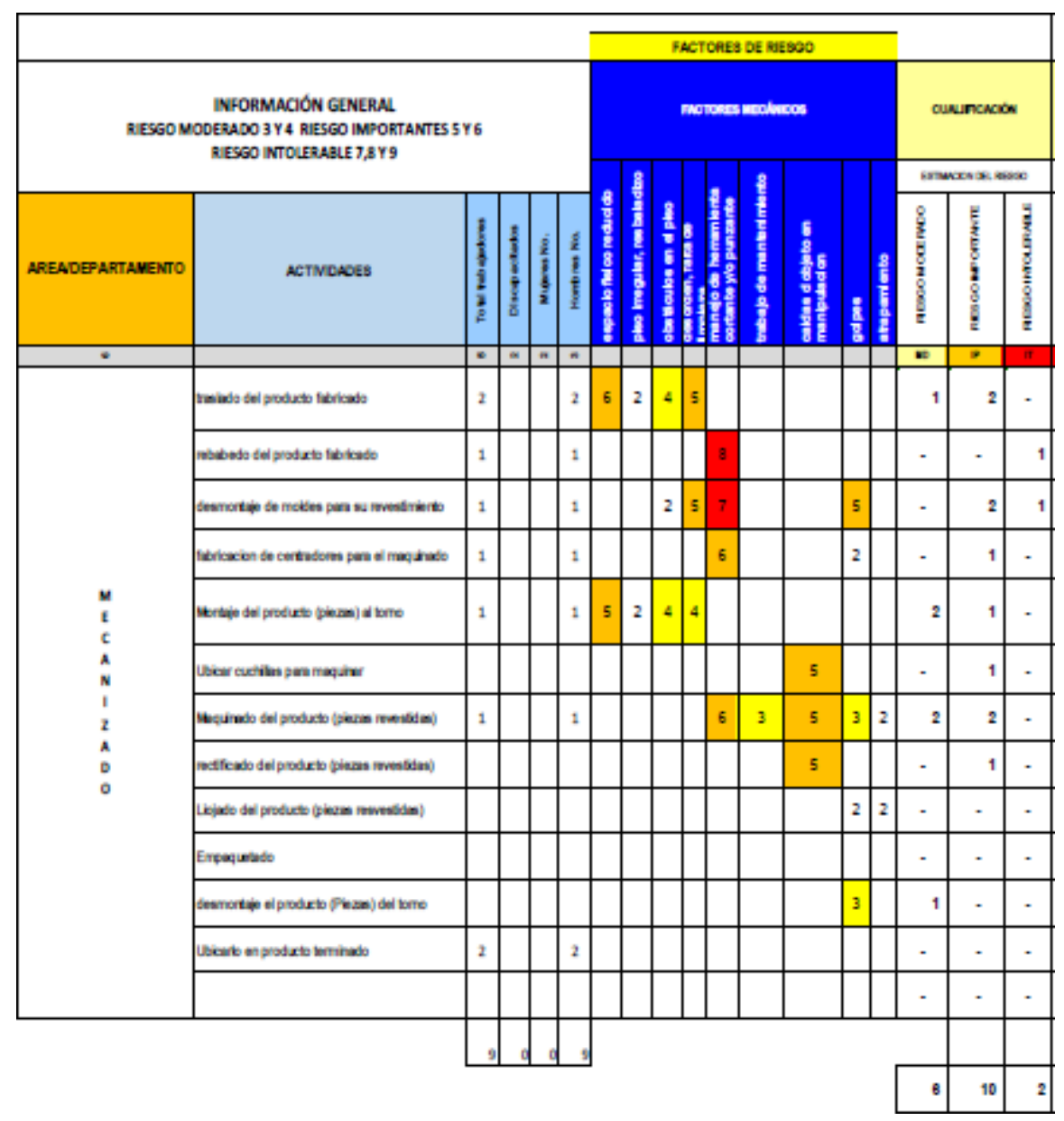

Se analizan los riesgos mecánicos en las actividades realizadas en el área de mecanizado donde resaltaron seis riegos moderados, 9 riegos importantes y 3 riegos intolerables. Los riesgos mecánicos de acuerdo con el análisis realizado en el área de mecanizado dan como resultado mayor 
Evaluación de riesgos mecánicos en área de mecanizado con método fine para prevenir accidentes

riesgos en las actividades realizadas en que hay que tomar inmediatamente medidas preventivas para eliminar o reducir los riesgos analizados en la matriz de riesgos elaborada.

\section{Riesgos Químicos}

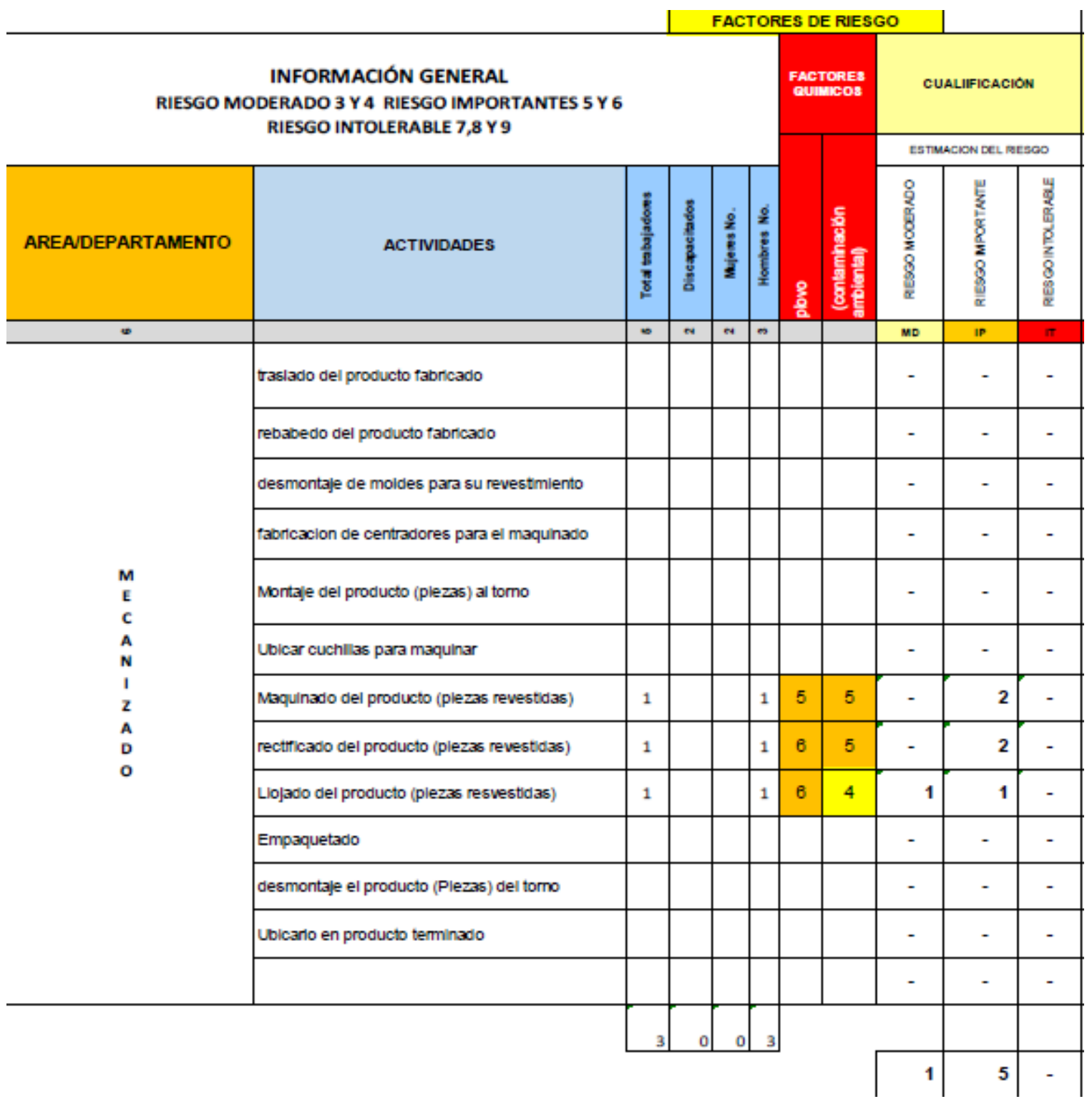

Se analizaron los riesgos químicos en las actividades realizadas en el área de mecanizado donde resaltaron un riesgo moderado y 5 riesgos importantes debido al polvo al rectificar los trabajos fabricados por la empresa donde se debe tomar en cuenta medidas para corregir las 
actividades que ocasionan este incidente en el puesto de trabajo, y salvaguardar la salud y la garantizar la seguridad de los trabajadores del área de mecanizado de la empresa, para lograrlo se debe tener en cuenta factores sumamente importantes que se verán más adelante.

\section{Riesgos Biológicos}

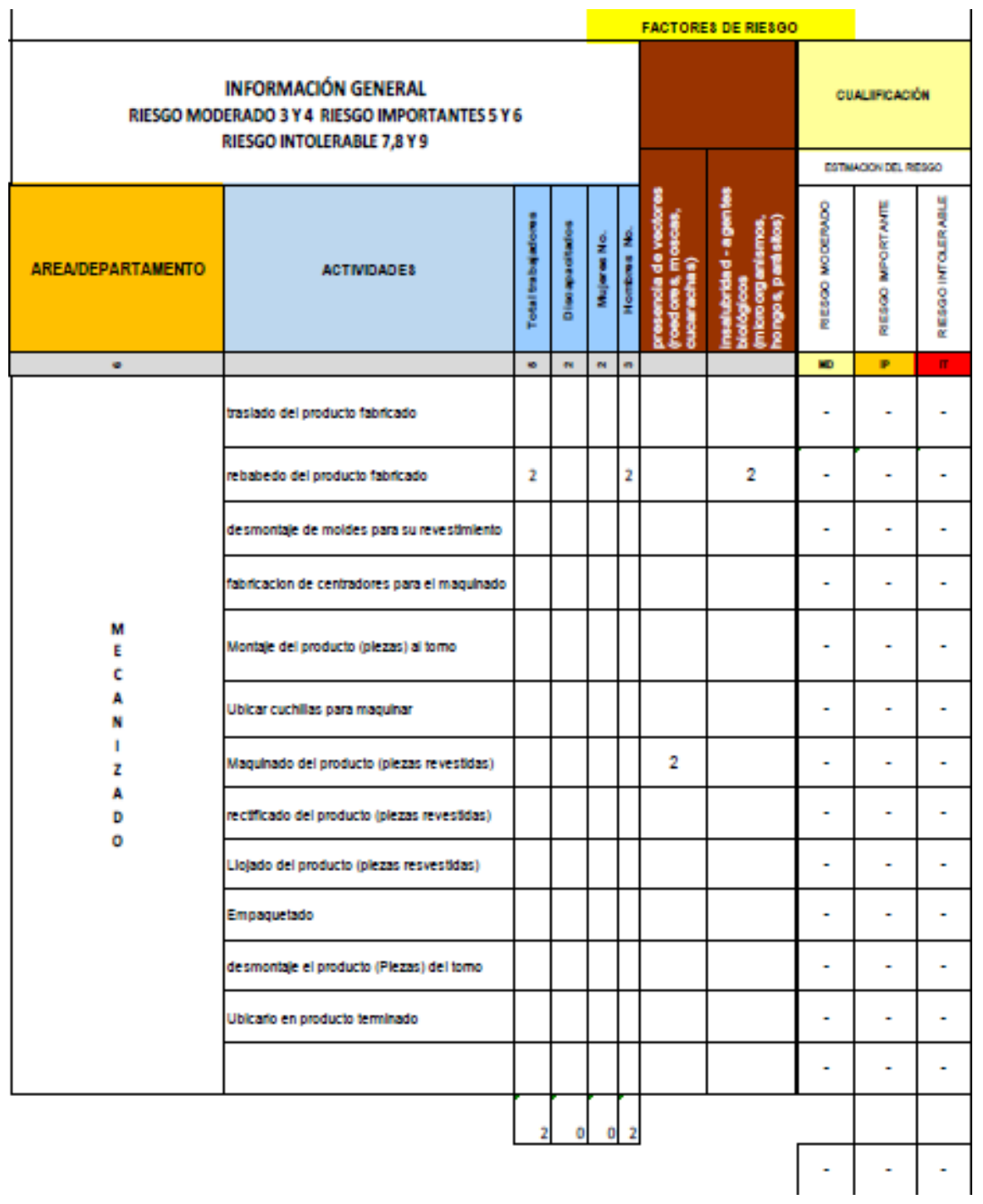

Se analizaron riesgos biológicos tales como presencia de vectores, insalubridad como la presencia de agentes biológicos en las actividades realizadas en el área de mecanizado donde 
Evaluación de riesgos mecánicos en área de mecanizado con método fine para prevenir accidentes

resaltaron riesgos tolerables que no es necesario tomar acciones preventivas sin embargo se debe considerar soluciones a las actividades realizadas en el puesto de trabajo.

\section{Riesgos Ergonómicos}

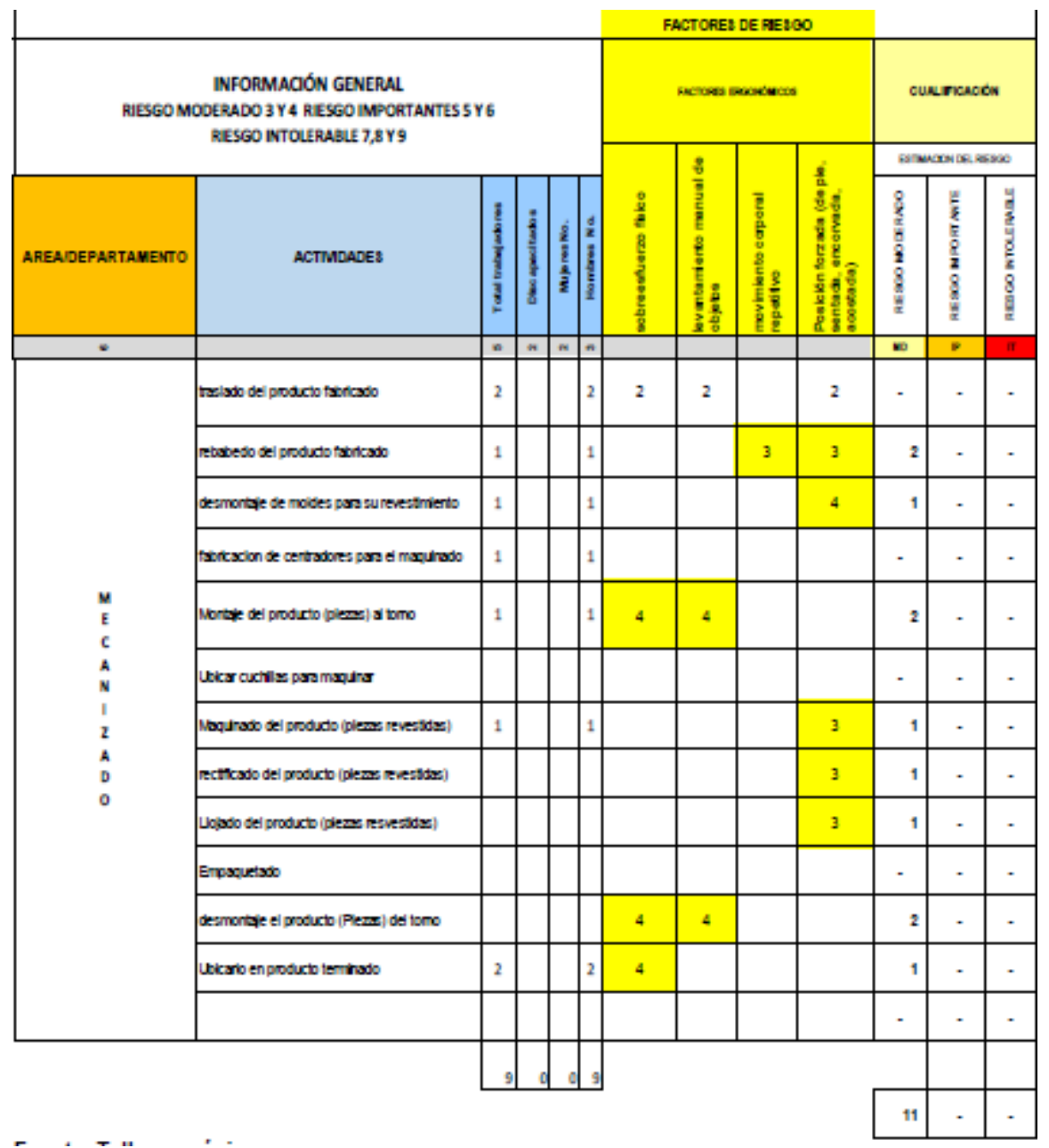

Se analizaron los riesgos ergonómicos en las actividades realizadas en el área de mecanizado donde resaltaron once riesgos moderado debido a la posición de los trabajadores a realizar sus actividades de acuerdo con el análisis realizado con valorización a nivel del riesgo se deberá hacer un esfuerzo para reducir los riesgo en el puesto de trabajo. 


\section{Riesgos Psicosociales}

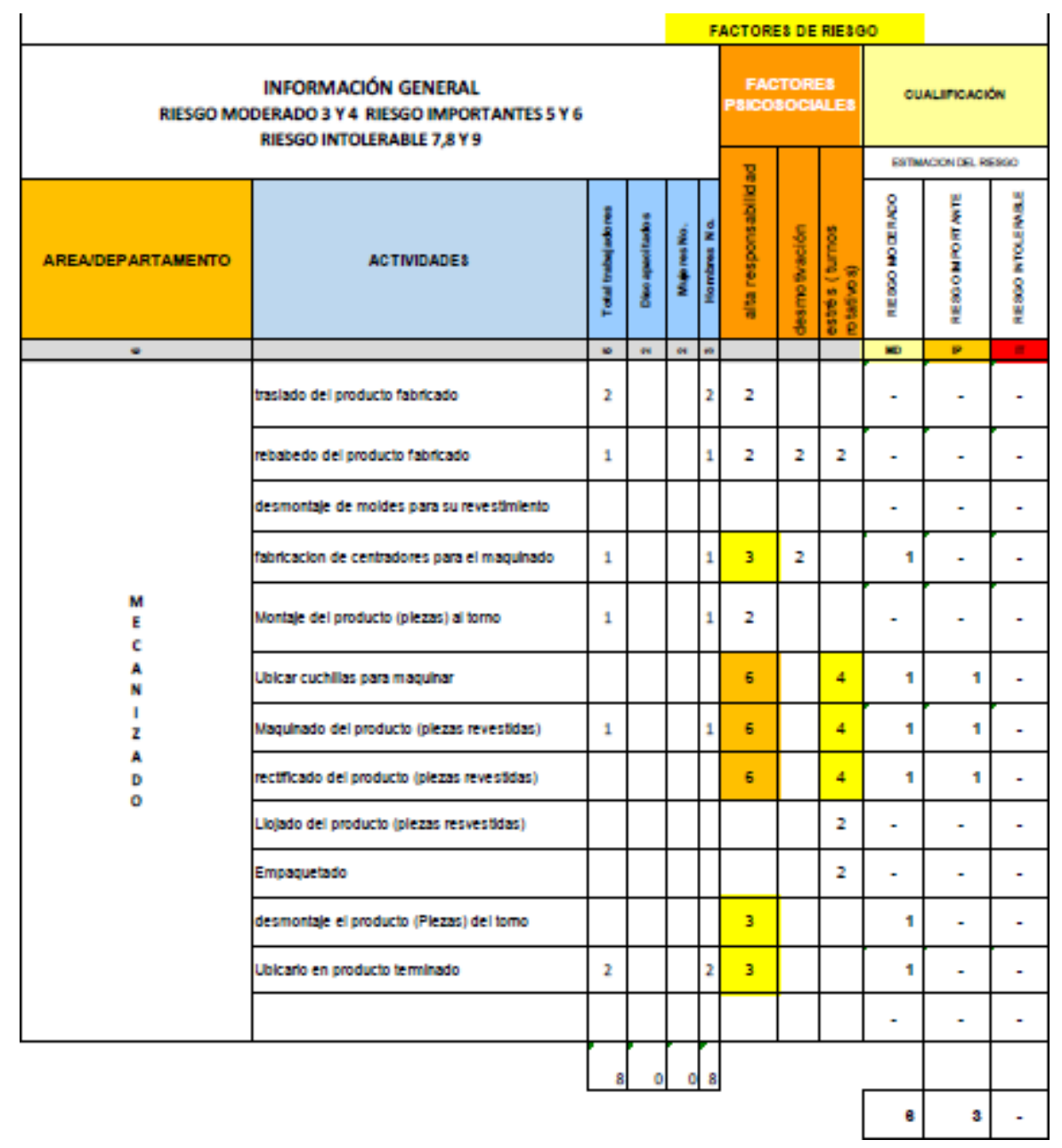

Se analizaron los riesgos psicosociales en las actividades realizadas en el área de mecanizado donde resaltaron seis riesgos moderados y tres riesgos importantes debido a la responsabilidad que tienen a realizar su trabajo y a turnos rotativos se debe tomar medidas preventivas para corregir las actividades analizadas en el puesto de trabajo de todos los colaboradores del área.

\section{Conclusiones.}

Después de haber realizado la evaluación investigativa en el área de mecanizado en las actividades realizadas se toman las siguientes conclusiones: que en la empresa se debe de elaborar e 
implementar las propuestas las cuales incluyen la capacitación al personal sobre la prevención de riesgos laborales y demás temas que contiene las capacitaciones como el buen manejo del EPP (equipo de protección personal) y la compra de los equipos de protección personal de acuerdo con las actividades realizadas.

Con las propuestas hechas se pretende reducir los riesgos mecánicos en el área de mecanizado más aún los riesgos de cortes que resaltaron con mayor peligro en la matriz triple criterio, cabe recalcar que la inversión de la alternativa propuesta es factible de acuerdo con el método de W. FINE.

\section{Bibliografia.}

Beltrán Molina, J., López Becerra, L., \& Murcia Chinchilla, Y. (2015). Condiciones de seguridad en el trabajo relacionadas con la exposición a peligro mecánico. Revista de la Universidad Industrial de Santander. Salud, 47(2), 193-198.

Díaz Moline, R. (2007). Guía práctica para la prevención de riesgos laborales (5 ed.). México: Editorial Lex Nova.

DÍAZ ZAZO, M. (2015). Prevención de riesgos laborales. Seguridad y salud laboral. Madrid: Paraninfo.

Ruiz Hernández , V. (2011). GUÍA PARA EL MANTENIMIENTO Y PRÁCTICAS DE LABORATORIO PARA TORNOS DE FILETEAR KENT-USA 1340A UTILIZADOS PARA EL LABORATORIO DE PROCESOS DE MANUFACTURA 1. Guatemala: Universidad de San Carlos de Guatemala.

Ulloa-Enríquez, M. (2012). Riesgos del Trabajo en el Sistema de Gestión de Calidad. Ingeniería Industrial, 33(2), 100-110. 